\title{
UNE APPROCHE HYBRIDE POUR LE SAC À DOS MULTIDIMENSIONNEL EN VARIABLES 0-1
}

\author{
Michel VAsquez $^{1}$ et Jin-KaO HaO ${ }^{2}$ \\ Communiqué par Gérard Plateau
}

\begin{abstract}
We present, in this article, a hybrid approach for solving the $0-1$ multidimensional knapsack problem (MKP). This approach combines linear programming and Tabu search. The resulting algorithm improves on the best result on many well-known hard benchmarks.
\end{abstract}

Résumé. Nous présentons, dans cet article, une approche hybride pour la résolution du sac à dos multidimensionnel en variables 0-1. Cette approche combine la programmation linéaire et la méthode tabou. L'algorithme ainsi obtenu améliore de manière significative les meilleurs résultats connus sur des instances jugées difficiles.

Mots clés : Sac-à-dos multidimensionnel, programmation linéaire, recherche tabou.

\section{INTRODUCTION}

Le sac à dos multidimensionnel en variables bivalentes (MKP01) permet de modéliser une grande variété de problèmes où il s'agit de maximiser un profit tout en ne disposant que de ressources limitées. Son expression formelle est la suivante :

$$
\operatorname{MKP} 01\left\{\begin{array}{l}
\text { maximiser } \text { c. } x \\
\text { s.c. } A . x \leq b \text { et } x \in\{0,1\}^{n}
\end{array}\right.
$$

Reçu en mars 2000.

${ }^{1}$ LGI2P, Parc Scientifique Georges Besse, 30035 Nimes Cedex 1, France;

e-mail : vasquez@site-eerie.ema.fr

2 LERIA, Université d'Angers, 2 bd Lavoisier, 49045 Angers Cedex 1, France;

e-mail : Jin-Kao.Hao@univ-angers.fr 
avec $c \in \mathbb{N}^{* n}, A \in \mathbb{N}^{m \times n}$ et $b \in \mathbb{N}^{m}$. Les coordonnées binaires $x_{j}$ du vecteur $x$ sont des variables de décision : $x_{j}=1$ si l'objet $j$ est retenu dans le sac, 0 sinon. $c_{j}$ est le profit (ou gain) associé à l'objet $j$. Les éléments $\left(a_{i j}\right)$ de la matrice $A$ représentent, pour chaque type de ressource $i$, la consommation de l'objet $j$. Enfin les $b_{i}$ sont les quantités disponibles de chacune des $m$ ressources. L'objectif est de trouver un sous ensemble d'objets qui maximise le profit tout en respectant les contraintes de limitation de ressources.

Le fait qu'on le rencontre dans des domaines d'application aussi différents que l'économie [25], l'industrie [9, 18,32], les transports, le chargement de cargaison $[4,31]$ et l'informatique répartie [16], etc. lui confère un grand intérêt pratique. Ce problème d'optimisation combinatoire sous contraintes est cependant NP-difficile [15]. Sa résolution revêt donc, aussi, un caractère théorique toujours d'actualité.

Ainsi de nombreux travaux ont été effectués sur le sujet $[1,2,5,7,8,10,11,17,20$, $23,24,31,32]$ et beaucoup d'heuristiques sont encore explorées pour proposer des solutions, sinon exactes, du moins approchées à ce problème. En effet les méthodes exactes sont limitées à de petites instances ou à des instances ayant des caractéristiques bien spécifiques : matrice $A$ creuse et quasi unimodulaire, redondance de contraintes etc. Aujourd'hui, la résolution des instances les plus difficiles de MKP01 se réalise donc essentiellement avec des approches heuristiques. L'aptitude de ces dernières à fournir des solutions de bonne qualité les rend indispensables dans le domaine pratique. Elles s'avèrent aussi très utiles pour le développement d'algorithmes exacts fondés sur des méthodes d'évaluation et séparation.

Nous proposons, dans ce papier, une approche hybride qui combine la programmation linéaire et la recherche locale tabou. L'idée fondamentale de notre approche consiste à explorer l'espace de recherche, d'une manière contrôlée, autour d'optima continus de problèmes relaxés. Ainsi nous nous distinguons des approches fondées sur le critère de choix classique du ratio profit/ressource. Pour mettre en ouvre cette approche, nous proposons d'abord une méthode permettant de déterminer le nombre d'objets à l'optimum de MKP01. Nous développons ensuite un algorithme de recherche locale intégrant un voisinage original et une implémentation efficace de la technique d'élimination inverse pour la gestion de la liste tabou.

Nous validons cette approche sur des benchmarks classiques ainsi que des benchmarks récents réputés difficiles $[7,20]$. Nous montrons que cette approche permet non seulement de retrouver les meilleurs résultats connus pour l'ensemble des jeux expérimentés, mais aussi d'améliorer de manière significative les meilleurs résultats pour un grand nombre de jeux les plus difficiles [7,23].

Notre exposé s'organise comme suit. Après un état de l'art sur les principales stratégies de résolution (Sect. 2), nous donnons le principe général de notre approche hybride (Sect. 3). Nous développons ensuite la phase de la programmation 
linéaire (Sect. 4), ainsi que la phase de la recherche locale tabou (Sect. 5). Ces développements sont alimentés par la présentation des résultats expérimentaux (Sect. 6), ainsi qu'une analyse sur les performances de notre algorithme (Sect. 7). Nous terminons par une discussion sur de nombreuses perspectives.

\section{Stratégies DE RÉSOLUTION}

Afin de situer notre approche dans le contexte général des méthodes de résolution, nous présentons ici, quelques uns des axes de recherche explorés.

\subsection{MÉThodes EXACTES}

Il existe un algorithme pseudo polynômial pour résoudre le MKP01 par la programmation dynamique $[4,21]$. Sa complexité $O\left(n \prod_{i=1}^{m}\left(b_{i}+1\right)\right)$ le rend très vite impraticable en fonction de $m$ et des $b_{i}$.

Les algorithmes d'évaluation et séparation se distinguent essentiellement par le calcul de borne qu'ils mettent en œuvre pour élaguer l'arbre de recherche. Un premier algorithme de branch \& bound [31] propose de prendre, comme borne supérieure pour l'évaluation, la valeur $\min \left\{z_{1}(k), \ldots, z_{m}(k)\right\}$ où $z_{i}(k)$ est l'optimum du sac à dos ne contenant que la $i$ ème contrainte au nœud $k$ de l'arborescence. La variable de branchement étant alors celle qui correspond à l'élément fractionnaire de ce problème de sac à dos unidimensionnel. L'avantage de cette méthode est qu'elle ne traite que des sacs à dos simples que l'on sait bien résoudre [28]. En revanche la borne utilisée est assez imprécise. Dans l'exemple suivant :

$$
\text { exemple } 1(n=4, m=2)\left\{\begin{array}{l}
c=\left(\begin{array}{llll}
4 & 5 & 4 & 4
\end{array}\right) \\
A=\left(\begin{array}{llll}
1 & 3 & 3 & 2 \\
3 & 3 & 2 & 1
\end{array}\right) \quad b=\left(\begin{array}{l}
3 \\
3
\end{array}\right)
\end{array}\right.
$$

$z_{1}=8$ pour $x_{1}=(1,0,0,1)$ et $z_{2}=8$ pour $x_{2}=(0,0,1,1)$, alors que la solution optimale qui tient compte des deux contraintes, est $\bar{x}=(0,1,0,0)$ et vaut $\bar{z}=5$. Non seulement $\bar{z}$ est relativement loin de $\min \left\{z_{1}, z_{2}\right\}$, mais l'information sur les composantes de $\bar{x}$ est complètement fausse.

L'estimation, plus élaborée, de la valeur potentielle d'un branchement à partir de multiples relaxations (lagrangienne, composite...) et du calcul des multiplicateurs duaux [17] donne de meilleurs résultats que le précédent algorithme avec toutefois un accroissement sensible de la complexité de calcul. D'une manière générale les algorithmes d'énumération implicite se heurtent à une combinatoire en $O\left(2^{n}\right)$. Pour élargir leur domaine d'application à des instances de taille plus grande on peut d'une part chercher de bons minorants par des heuristiques de construction efficaces, et d'autre part réduire la taille du problème par fixation de variables [21], élimination de contraintes [11] ou encadrement du nombre de 
variables à l'optimum [12]. Ces dernières méthodes font appels aux aspects les plus théoriques de la programmation linéaire.

\subsection{MÉThOdes APPROCHÉES}

Plusieurs algorithmes approchés combinent la programmation linéaire avec une heuristique dont l'objectif est de rendre entières des variables continues. En effet, pour utiliser la programmation linéaire il faut supprimer le caractère binaire des variables $x_{j}$. La relaxation des contraintes d'intégrité est une technique omniprésente dans les méthodes de résolution de MKP01. Elle conduit à la résolution du problème associé à MKP01 suivant :

$$
\operatorname{MKP}\left\{\begin{array}{l}
\text { maximiser } c . x \\
\text { s.c. } A . x \leq b \text { et } x \in[0-1]^{n} .
\end{array}\right.
$$

Les composantes du vecteur $x$ sont, dans le cas de MKP, comprises dans l'intervalle continu [0-1]. MKP se résout efficacement ${ }^{3}$ par la méthode du simplexe [30].

La résolution de MKP fournit une borne supérieure $\bar{z}$ qui, associée aux coûts réduits des variables hors base, permet d'introduire une technique de fixation de variables [21]. Une des techniques consiste à faire entrer les variables d'écart en base [2]. Ce principe a été repris et intégré à des approches heuristiques [1,24]. Enfin, L $\phi$ kketangen et Glover ont réalisé plusieurs implantation d'algorithmes tabou exploitant le tableau du simplexe $[26,27]$.

Par ailleurs on retrouve les valeurs des variables duales à l'optimum de MKP comme multiplicateur $u$ du vecteur colonne $a_{j}$ des consommations de l'objet $j$, dans les critères de type ratio $c_{j} /\left(u \cdot a_{j}\right)$, pour le choix d'une variable $x_{j}$. Ce ratio profit/ressource intervient, sous différentes variantes, dans la majorité des heuristiques fondées sur des mécanismes de transformation locale de la solution courante $[7,8,20,23]$.

Parmi ces dernières nous soulignons les algorithmes génétique (AG) de Chu et Beasley [7] et tabou de Hanafi et Fréville [23] qui obtiennent les résultats parmi les meilleurs sur des instances difficiles de MKP01. Ces deux algorithmes se distinguent par la métaheuristique mise en œuvre pour contrôler l'exploration de l'espace de recherche (AG/tabou) ainsi que par une petite variante dans le mécanisme de transformation des configurations $x$ visitées par le processus d'optimisation. Cette opération correspond à l'algorithme bien connu DROP-ADD suivant :

\footnotetext{
${ }^{3}$ C'est ici l'aspect pratique qui nous intéresse, il est vrai qu'en théorie le comportement du simplexe peut être exponentiel.
} 
Algorithme 1 : DROP-ADD

\section{Enlever:}

\begin{tabular}{l|l}
{$[1]$} & $i^{*}=\arg \max \left\{\left(\sum_{j=1}^{n} a_{i j} . x_{j}\right) / b_{i}, i \in[1, m]\right\}$ \\
{$[2]$} & $j^{*}=\arg \min \left\{c_{j} / a_{i^{*} j} \mid x_{j}=1, j \in[1, n]\right\}$ \\
& $x_{j^{*}} \leftarrow 0$
\end{tabular}

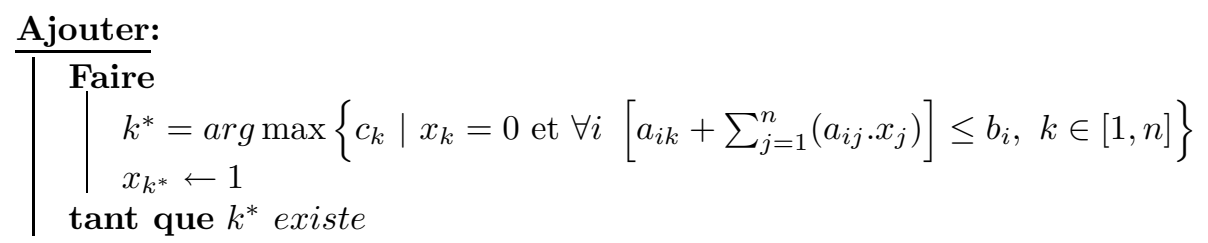

Les lignes 1 et 2 se retrouvent dans l'algorithme [23] (ainsi que dans [8]...). La ligne 1 identifie la contrainte la plus saturée. C'est une façon de s'affranchir du calcul du vecteur multiplicateur $u$ tout en conservant le critère du ratio profit/ressource. Dans [7] cette technique est remplacée par

$$
j^{*}=\arg \min \left\{c_{j} /\left(u . a_{j}\right) \mid x_{j}=1, j \in[1, n]\right\}
$$

où $u$ correspond aux coûts marginaux des $m$ ressources de MKP.

Considérons cependant l'instance de MKP01 suivante :

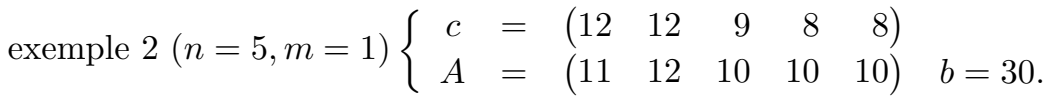

Un algorithme glouton guidé par l'heuristique du ratio profit/ressource (ou simplement celle du meilleur profit) pour ajouter des objets dans le sac construira la solution $x=(1,1,0,0,0)$ qui vaut $z=24$ alors que la solution binaire optimale est $\dot{x}=(0,0,1,1,1)$ pour $\dot{z}=25$.

Cet exemple n'est pas une démonstration, mais il illustre l'hypothèse suivante. Le rôle de la transformation locale est mineur devant celui de la stratégie globale d'exploration de l'espace de recherche. Cette vision globale est naturelle pour un algorithme génétique ; elle correspond à la population de vecteurs $x$ impliqués par les opérateurs de croisement, de sélection et de mutation, qui caractérisent cette métaheuristique (une centaine d'individus sont gérés simultanément dans [7]). Elle n'est pas systématique pour un algorithme tabou. Elle correspond, dans ce cas, à une alternance de phases d'intensification et de diversification de la recherche. Dans [23] cette alternance de phases est renforcée par une stratégie d'oscillations entre la zone de faisabilité $(A . x \leq b)$ et la zone d'infaisabilité $\left(\exists i \in[1, m] \mid a_{i} . x>b_{i}\right)$. 
Pour finir ce survol des métaheuristiques appliquées à MKP01, nous citerons le recuit simulé [10], ainsi qu'une étude des méthodes de bruitage [5] fondées sur les travaux de Charon et Hudry [6]. Les références [5,10] ne présentent cependant que des résultats sur des instances de petites tailles. Enfin, Fréville et Plateau ont proposé une heuristique, qui combine des calculs de coefficients duaux avec des fixations de variables, une élimination de contraintes et une procédure d'énumération, pour résoudre efficacement des instances à 2 contraintes [13].

\section{Principe GÉNÉRAL DE NOtRe ALGORIThme}

Tenant compte des remarques faites sur l'exemple 2 de la section 2.2 , notre étude sur la résolution approchée de MKP01 par la recherche locale $(R L)$ s'est plus particulièrement focalisée sur les deux points suivants :

1. remise en cause de l'heuristique de mouvement (ou critère de choix d'un voisin de la configuration courante) ;

2. recherche d'un moyen d'enrichir le simple processus de $R L$ par une stratégie globale.

Nous avons trouvé un principe commun pour répondre à ces deux points d'investigation.

L'idée maîtresse de notre approche est de chercher autour de l'optimum $\bar{x}$ de MKP. Notre hypothèse est que les points binaires proches de $\bar{x}$ sont de bonne qualité. Le vecteur $\bar{x}$ contient l'information globale qui guidera notre processus de $R L$ tout en contrôlant le mécanisme de visite du voisinage $\mathcal{N}(x)$ d'une configuration courante $x$. Pour ce faire nous limitons la $R L$ aux seuls points $x^{\prime} \in \mathcal{S}$ tels que : distance $\left(x^{\prime}, \bar{x}\right) \leq \delta_{\max }{ }^{4}$. La distance géométrique intervient dans le mécanisme de mouvement. Le vecteur ou point $\bar{x}$ peut être considéré comme une information à caractère stratégique pour la recherche locale.

Sur l'exemple 2 (Sect. 2.2) le simplexe donne $\bar{z}=30.3$ et $\bar{x}=\left(1,1, \frac{7}{10}, 0,0\right)$ alors que, rappelons-le, $\dot{z}=25$ pour $\dot{x}=(0,0,1,1,1)$. De ce point de vue notre heuristique n'est donc pas plus convaincante que celle du ratio profit/ressource. Enfin si l'on peut considérer $\bar{x}$ comme un élément de connaissance à apporter au processus de $R L$, son unicité en réduit passablement le caractère global.

Faisons pourtant la remarque suivante : toute(s) solution(s) de MKP01 vérifie(nt) l'égalité : $\sum_{j=1}^{n} x_{j}=k \in \mathbb{N}$. Si nous ajoutons cette égalité comme contrainte au problème MKP nous obtenons une série de problèmes du type :

$$
\operatorname{MKP}[k]\left\{\begin{array}{l}
\text { maximiser } c . x \\
\text { s.c. } A . x \leq b \text { et } x \in[0-1]^{n}, \\
\sigma(x)=k \in \mathbb{N}
\end{array}\right.
$$

où $\sigma(x)$ est la somme des composantes du vecteur $x$. Nous disposons donc de plusieurs points $\bar{x}_{[k]}$ pour explorer l'espace de recherche $\mathcal{S}$. Cette approche, directement inspirée par les travaux de Fréville et Plateau [12], n'est pas isolée. En

\footnotetext{
${ }^{4}$ Nous préciserons en section 5.2 les notions distance et $\delta_{\max }$.
} 
effet, de manière très contemporaine, l'ajout de la contrainte d'égalité a été mis en œuvre pour réduire l'espace de recherche dans le cas du sac à dos multidimensionnel multiobjectif [14]. Illustrons cette heuristique sur ce même exemple de la section 2.2 .

$$
\begin{aligned}
& \mathrm{MKP}[1] \rightarrow \bar{z}_{[1]}=12 \quad \text { et } \quad \bar{x}_{[1]}=(1,0,0,0,0) \\
& \mathrm{MKP}[2] \rightarrow \bar{z}_{[2]}=24 \quad \text { et } \quad \bar{x}_{[2]}=(1,1,0,0,0) \\
& \mathrm{MKP}[3] \rightarrow \bar{z}_{[3]}=25 \quad \text { et } \quad \bar{x}_{[3]}=(0,0,1,1,1) \text {. }
\end{aligned}
$$

Son comportement semble plus prometteur et nous pouvons énoncer l'idée générale de notre algorithme hybride : dans chaque hyperplan $\sigma(x)=k$ lancer un processus de $R L$ autour du point $\bar{x}_{[k]}$. Nous allons donc alterner, dans le processus d'optimisation, une phase de programmation linéaire $P L$ avec une phase de recherche locale tabou $R L^{\text {tabou }}$. Chaque séquence $P L / R L^{\text {tabou }}$ pourra être distribuée sur plusieurs machines.

La mise en œuvre de ce principe se décompose en trois étapes :

- détermination des valeurs de $k$ intéressantes ;

- utilisation du simplexe pour calculer les $\bar{x}_{[k]}$ correspondants ;

- exécution de la recherche locale tabou autour de ces points.

La section suivante décrit la phase simplexe, ou programmation linéaire $P L$, qui traite les deux premières étapes que nous venons de citer.

\section{Phase Simplexe}

Le processus $R L^{\text {tabou }}$ effectue un échantillonnage discret autour de l'optimum de $\operatorname{MKP}[k]$. Notre hypothèse est qu'en tant que tel il ne fera pas mieux que la valeur $\bar{z}_{[k]}$ de la solution continue de ce programme. La première étape de notre approche consiste donc à trouver les valeurs de $k$ qui fournissent des bornes supérieures $\bar{z}_{[k]}$ à un minorant $z$ donné.

Fréville et Plateau [12] proposent un algorithme pour l'encadrement du nombre de variables à l'optimum de MKP01 qui fait appel à plusieurs outils de la programmation linéaire. Nous avons développé un algorithme plus simple dont le principe est d'utiliser $R L^{\text {tabou }}$ pour obtenir un minorant $z$, puis résoudre par le simplexe les deux problèmes suivants $\operatorname{MKP} \sigma \min [z]$ et $\operatorname{MKP} \sigma \max [z]$ :

$$
\operatorname{MKP} \sigma \min [z]\left\{\begin{array}{l}
\text { minimiser } \sigma(x) \\
\text { s.c. } A . x \leq b \text { et } x \in[0-1]^{n}, \\
\text { c. } x \geq(z+1)
\end{array}\right.
$$


Soit $\sigma \min [z]$ la valeur optimale de ce problème. Si nous prenons moins d'objets que $k_{\min }=\lceil\sigma \min \rceil$ la contrainte $c . x \geq(z+1)$ ne sera plus vérifiée.

$$
\operatorname{MKP} \sigma \max [z]\left\{\begin{array}{l}
\text { maximiser } \sigma(x) \\
\text { s.c. } A . x \leq b \text { et } x \in[0-1]^{n}, \\
\text { c. } x \geq(z+1)
\end{array}\right.
$$

soit $\sigma \max [z]$ la valeur optimale de ce problème. Si nous prenons plus d'objets que $k_{\max }=\lfloor\sigma \max \rfloor$ l'une au moins des contraintes $A . x \leq b$ ne sera plus vérifiée. En conséquence, les seules valeurs de $k$ intéressantes pour notre processus de recherche locale sont celles comprises entre $k_{\min }$ et $k_{\max }$. Nous calculons donc, par l'algorithme du simplexe, les $\left(k_{\max }-k_{\min }+1\right) \operatorname{MKP}[k]$ qui nous fournissent les points cherchés pour la phase $R L^{\text {tabou }}$.

La figure 1 résume le déroulement de cette phase simplexe ou $P L$ sur l'instance CB30.250.10 due à Chu et Beasley [7]. Le programme $R L^{\text {tabou }}$ a besoin d'un point pour amorcer la recherche, cela explique la ligne 1 de cet exemple.

1. résoudre $\mathrm{MKP} \rightarrow \bar{x}, \bar{z}=108258.07$ et $\sigma(\bar{x})=125.65$,

2. lancer $R L^{\text {tabou }}$ autour de $\bar{x} \rightarrow z=107611$,

3. $\sigma \min [z]=122.17 \rightarrow k_{\min }=123$,

4. $\sigma \max [z]=128.56 \rightarrow k_{\max }=128$,

5 .

\begin{tabular}{|c|c|c|c|c|c|c|}
\hline$k$ & 123 & 124 & 125 & 126 & 127 & 128 \\
\hline $\bar{z}_{[k]}$ & 107811.87 & 108055.23 & 108212.76 & $\mathbf{1 0 8 2 4 8 . 6 2}$ & 108138.39 & 107802.92 \\
\hline
\end{tabular}

Figure 1. Phase $P L$ pour cB30.250.10.

Remarquons enfin que la meilleure valeur $\bar{z}_{[k]}=108248.62$ obtenue par les $\left(k_{\max }-k_{\min }+1\right) \operatorname{MKP}[k]$ est plus fine, en tant que borne supérieure de MKP01, que $\bar{z}=108258.07$ produite par la résolution de MKP.

\section{Phase ReCherche locale}

\subsection{DÉFinitions}

Avant de détailler notre algorithme tabou nous résumons, dans ce paragraphe, les éléments de terminologie que le lecteur retrouvera par la suite :

- la configuration $x$ : c'est le vecteur binaire $\left(x_{1}, \cdots, x_{n}\right)$;

- $\mathcal{S}$ représente l'espace de recherche. Il peut être égal à $\{0,1\}^{n}$ si l'on considère toutes les configurations possibles $x \in \mathcal{S}$ réalisables ou non ;

- le voisinage de $x: \mathcal{N}(x)$ est le sous-ensemble des éléments de $\mathcal{S}$ accessibles depuis l'élément $x$ en une seule itération ; 
- l'attribut est la valeur affectée à une composante. Dans le cas binaire on peut identifier sans ambiguïté un attribut à l'indice $j$ de la composante qui change de valeur ;

- enfin le mouvement correspond au passage de la $R L$ du point $x$ à un de ces voisins $x^{\prime}: \operatorname{mvt}\left(x, x^{\prime}\right) \mid x^{\prime} \in \mathcal{N}(x)$. En reprenant ce qui vient d'être dit sur les attributs on peut aussi écrire $m v t\left(i_{1}, i_{2}, \ldots, i_{k}\right)$ où les indices $i_{j}$ sont ceux des composantes de $x$ qui sont complémentées dans $x^{\prime}$. Un tel mouvement est un $k_{-}$change.

\subsection{RÉduction DE L’espace DE RECHERChe}

Nous allons dans cette section spécifier un sous ensemble $\mathcal{X} \subset \mathcal{S}$ dans lequel naviguera notre processus de recherche locale. Cette réduction de $\mathcal{S}$ reprend les idées que l'on vient d'énoncer :

1. limitation de $\mathcal{S}$ à une sphère d'un rayon fixé autour du point $\bar{x}_{[k]}$ solution optimale de $\operatorname{MKP}[k]$;

2. conservation du nombre d'objets retenus dans les configurations $x$, produites par $R L^{\text {tabou }}$ autour du point $\bar{x}_{[k]}$, à la valeur constante $k$ (intersection de $\mathcal{S}$ avec l'hyperplan $\{\sigma(x)=k\})$.

Pour le point 1 nous utilisons la distance $\delta$ définie, pour $x$ et $x^{\prime}$ binaires ou continus, par la formule $\delta\left(x, x^{\prime}\right)=\sum_{j=1}^{n}\left|x_{j}-x_{j}^{\prime}\right|$. L'heuristique pour estimer la distance maximale $\delta_{\max }$ autorisée depuis le point $\bar{x}_{[k]}$, est la suivante : soit $\left(1,1, \cdots, 1, r_{1}, \cdots, r_{q}, 0, \cdots, 0\right)$ les composantes triées par ordre décroissant du vecteur $\bar{x}_{[k]}$. Les $r_{j}$ sont les composantes fractionnaires de $\bar{x}_{[k]}$ et l'on a:

$$
1>r_{1} \geq r_{2} \geq \cdots \geq r_{q}>0 .
$$

Le mécanisme de $R L$ peut choisir, dans le pire des cas, les objets qui correspondent à ces composantes plutôt que ceux qui correspondent aux composantes à 1 . La figure 2 illustre cette configuration limite. $u$ est le nombre de composantes à 1 dans $\bar{x}_{[k]}, u+q$ est donc le nombre de composantes non nulles de $\bar{x}_{[k]}{ }^{5}$. De plus $\sigma\left(\bar{x}_{[k]}\right)=k \Rightarrow \sum_{j=1}^{q} r_{j}=k-u \Rightarrow \delta_{[k]}=2 \times(u+q-k) \in 2 \times \mathbb{N}$.

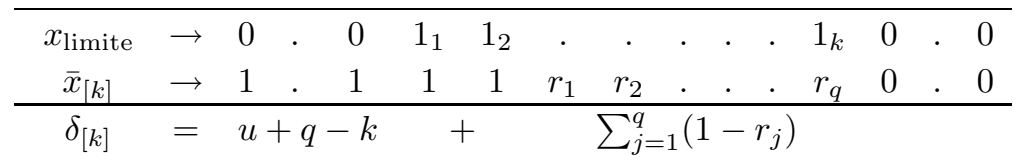

Figure 2. Heuristique de calcul de $\delta_{[k]}=\delta\left(x_{\text {limite }}, \bar{x}_{[k]}\right)$.

\footnotetext{
${ }^{5}$ Notons que $u<k<u+q$ sinon le problème est trivial.
} 
Dans le cas limite où $u=k$ nous obtenons $\delta_{[k]}=0$ ce qui est tout à fait logique puisque dans ce cas $\bar{x}_{[k]}$ est entièrement binaire. Pratiquement, et selon les instances, nous prendrons : $\delta_{\max } \approx \delta_{[k]}$.

Chaque processus $R L^{\text {tabou }}$ lancé autour de $\bar{x}_{[k]}$ a donc son propre espace de recherche $\mathcal{X}_{k}$ :

$$
\mathcal{X}_{k}=\left\{x \in\{0,1\}^{n} \mid \sigma(x)=k \wedge \delta\left(x, \bar{x}_{[k]}\right) \leq \delta_{\max }\right\} .
$$

Notons ici que les $\mathcal{X}_{k}$ sont disjoints. Cela réduit à 0 les risques de redondance d'exploration des processus $R L^{\text {tabou }}$ qui peuvent donc s'exécuter indépendamment.

\subsection{VoISINAGE}

L'ensemble $\mathcal{N}(x)$ des voisins d'une configuration $x$ est donc défini par la formule suivante :

$$
\mathcal{N}(x)=\left\{x^{\prime} \in \mathcal{X}_{k} \mid \delta\left(x, x^{\prime}\right)=2\right\}
$$

À cause de la contrainte $\sigma(x)=k$ (implicite dans $\left.\mathcal{X}_{k}\right), \mathcal{N}(x)$ n'est qu'un sousensemble des points à distance 2 de $x$. Sa cardinalité est :

$$
|\mathcal{N}(x)|=(n-k) \times k .
$$

Le mouvement induit par ce voisinage correspond au retrait d'un objet et l'ajout d'un autre, c'est un cas particulier de 2_change (cf. définitions Sect. 5.1). Nous noterons indifféremment $\operatorname{mvt}\left(x, x^{\prime}\right)$ et $\operatorname{mvt}(i, j)$ avec $x_{i}^{\prime}=1-x_{i}$ et $x_{j}^{\prime}=1-x_{j}$. Cette formulation du voisinage va être modifiée à la fin de la section suivante pour tenir compte d'éléments liés à la gestion de la liste tabou.

\subsection{Gestion Dynamique De la liste tabou}

La méthode d'élimination inverse ( $M E I)$, proposée par Glover [19], permet de définir le statut tabou d'un mouvement de manière exacte. Cela signifie qu'elle est équivalente à l'enregistrement complet des configurations visitées. Elle est qualifiée de liste stricte [3]. Son principe consiste à mémoriser dans une liste (running list) les attributs des mouvements effectués. Pour savoir si un nouveau mouvement est tabou il faut parcourir la running list à l'envers. Ce faisant on construit une autre liste, la séquence d'annulation résiduelle ou $S A R$ dans laquelle soit on recopie les attributs de la running list (y compris ceux du nouveau mouvement), s'ils n'y sont pas déjà, soit on les en enlève. Cette étape est appelée trace des attributs du mouvement. Si au cours de cette étape on rencontre la condition $S A R=\emptyset$ alors le nouveau mouvement nous ramène à un point déjà visité. Il faut donc le rendre tabou. La complexité de la $M E I$ est donc $O\left(\right.$ iter $\left.^{2}\right)$.

Dammeyer et Voß [8] ont réalisé une première mise en œuvre de la $M E I$ sur le MKP01, dans laquelle la $M E I$ analyse un nombre variable d'échanges causés 
par l'algorithme DROP-ADD. Ce voisinage nécessite la gestion de traces à nombre variable d'attributs. Cela accroît la complexité de la procédure. Dans le cas de notre voisinage à $k$ constant nous parcourons une seule fois la running list et chaque fois que $|S A R|=2$ nous rendons tabou le mouvement qui implique les attributs $S A R_{0}$ et $S A R_{1}$. L'algorithme suivant est associé à la proposition logique : $\operatorname{mvt}(i, j)$ tabou $\Leftrightarrow$ tabou $[i][j]=$ iter.

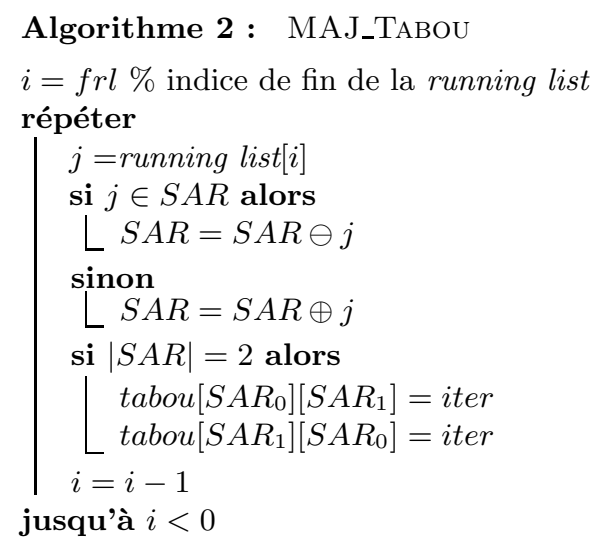

Le principe de la liste stricte peut provoquer le blocage de la $R L$ dans une impasse $x$, tous les points $x^{\prime} \in \mathcal{N}(x)$ ayant déjà été visités. La figure suivante illustre ce cas pour $\mathcal{S}=\{0,1\}^{4}$.

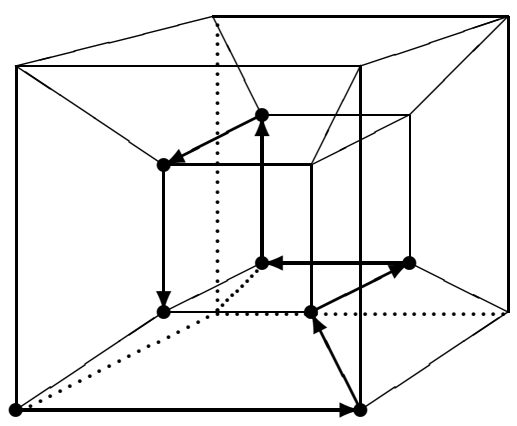

Figure 3. Blocage avec 1_change dans $\{0,1\}^{4}$.

En 7 itérations, avec un voisinage à 1_change, le chemin parcouru aboutit à une impasse alors que $\mathcal{S}$ n'a pas été complètement visité. Ce phénomène de blocage est d'une très faible probabilité dans $\{0,1\}^{n}$ avec $n \geq 100$. Nous ne l'introduisons que pour sensibiliser le lecteur à un aspect connexe beaucoup plus gênant : un processus de recherche locale contrôlé par une liste tabou stricte construit un chemin qui peut devenir une barrière entre $\mathcal{N}(x)$ et une zone de $\mathcal{X}_{k}$ de points potentiellement intéressants. Il faut donc autoriser, de temps en temps, le cyclage par un mécanisme d'aspiration [19,22]. 
Nous contournons cet écueil par une remise à zéro de la running list. Toutefois, pour que ce mécanisme n'entraîne pas un cyclage systématique, nous imposons que les configurations $x$ visitées après cette remise à zéro soient d'une valeur c. $x=z>z_{\min }$ où $z_{\min }$ représente la valeur de la meilleure configuration réalisable rencontrée jusque là. Évidemment pour que cela soit possible sans tomber dans le cadre d'une simple descente les configurations non réalisables $\left(\exists i \in[1, m] \mid a_{i} . x>b_{i}\right)$ sont admises.

Nous introduisons donc une mesure du niveau d'infaisabilité :

$$
v_{b}(x)=\sum_{i \mid a_{i} x>b_{i}}\left(a_{i} \cdot x-b_{i}\right)
$$

que nous chercherons à minimiser au cours de la $R L$. A chaque fois que $v_{b}(x)$ vaut 0 nous effaçons la running list et nous mettons à jour $z_{\min }$.

Voici, pour finir, la version définitive du voisinage :

$$
\mathcal{N}(x)=\left\{x^{\prime} \in \mathcal{X}_{k} \mid\left(\delta\left(x, x^{\prime}\right)=2\right) \wedge\left(c . x^{\prime}>z_{\min }\right) \wedge\left(\operatorname{mvt}\left(x, x^{\prime}\right) \text { non tabou }\right)\right\} .
$$

\subsection{Fonction D'Évaluation et heuristique DE MOUVEMENT}

Nous précisons, dans cette section, comment à partir d'un point $x$, nous choisissons parmi les points $x^{\prime} \in \mathcal{N}(x)$ le point $y$ qui fera l'objet du mouvement $\operatorname{mvt}(x, y)$.

La fonction d'évaluation d'une configuration $x$ a deux composantes : $v_{b}(x)$ et $z(x)=c . x$. La première composante est prioritaire sur la seconde. L'heuristique du choix d'un voisin de $x$ est donc :

$$
y=\left\{\begin{array}{l}
x^{\prime} \in \mathcal{N}(x) \mid \forall x^{\prime \prime} \in \mathcal{N}(x) \\
\left(v_{b}\left(x^{\prime}\right)<v_{b}\left(x^{\prime \prime}\right)\right) \vee\left(v_{b}\left(x^{\prime}\right)=v_{b}\left(x^{\prime \prime}\right) \wedge z\left(x^{\prime}\right) \geq z\left(x^{\prime \prime}\right)\right) .
\end{array}\right.
$$

En cas d'égalité de la fonction d'évaluation le choix est aléatoire.

\subsection{Configuration initiale}

Pour amorcer le processus $R L^{\text {tabou }}$ il faut construire un $x_{\text {init }}$ qui appartienne à $\mathcal{X}_{k}$. Nous allons montrer qu'il suffit, pour cela, de choisir les $k$ objets correspondant aux plus fortes composantes de $\bar{x}_{[k]}$, solution optimale de MKP[k] produit par simplexe.

Reprenons le schéma de la section 5.2 et construisons une suite de points $\left(x^{i}\right)^{6}$ qui part de $x_{\text {limite }}$ tout en décalant, à chaque étape, les $k$ composantes à 1 vers la gauche :

\footnotetext{
${ }^{6}$ Pour ne pas surcharger le texte nous avons utilisé la notation en exposant : $x^{i}$ représente un vecteur complet à ne pas confondre à la $i^{\text {ème }}$ composante $x_{i}$ du vecteur $x$.
} 


\begin{tabular}{cccccccccccccc}
\hline $\bar{x}_{[k]}$ & $\rightarrow$ & 1 &. & 1 & 1 & 1 & $r_{1}$ & $r_{2}$ &. &. &. & $r_{q}$ & 0 \\
\hline$x^{0}=x_{\text {limite }}$ & $\rightarrow$ & 0 &. & 0 & $1_{1}$ & $1_{2}$ &. &. &. &. &. & $1_{k}$ & 0 \\
$x^{1}$ & $\rightarrow$ & 0 &. & $1_{1}$ & $1_{2}$ &. &. &. &. &. & $1_{k}$ & 0 & 0 \\
$\ldots$ & $\rightarrow$ &. &. &. &. &. &. &. &. &. &. &. &. \\
$x_{\text {init }}=x^{u+q-k}$ & $\rightarrow$ & $1_{1}$ & $1_{2}$ &. &. &. &. &. & $1_{k}$ & 0 &. & 0 & 0 \\
\hline
\end{tabular}

Figure 4. Construction de $x_{\text {init }}$.

Comme on peut le voir sur la figure 4 la distance de $x_{1}$ à $\bar{x}_{[k]}$ est égale à celle de $x_{0}$ plus $\left(r_{q}-1\right)$ soit à $\delta_{[k]}+\left(r_{q}-1\right)$. Par définition $r_{q}$ est strictement inférieur à 1 donc $x_{1}$ s'est rapproché de $\bar{x}_{[k]}$. On a la relation de récurrence :

$$
\delta_{i}=\delta\left(x_{i}, \bar{x}_{[k]}\right)=\delta_{i-1}+\left(r_{q-i+1}-1\right)=\delta_{i-1}+\epsilon_{i} \text { avec } \forall i \epsilon_{i}<0
$$

La suite des distances $\left(\delta_{i}\right)$ correspondant à la suite $\left(x_{i}\right)$ est strictement décroissante depuis la valeur $\delta_{[k]}$ ce qui garantit : $\delta_{u+q-k}<\delta_{[k]}$. Nous pouvons prendre comme configuration initiale $x_{\text {init }}$ le point $x^{u+q-k}$ qui appartient bien à $\mathcal{X}_{k}$.

\subsection{Algorithme $R L^{\text {tabou }}$}

L'algorithme $R L^{\text {tabou }}$ (Algorithme 3) n'optimise pas directement $\mathrm{MKP}[k]$. Il résout $^{7}$ une suite de problèmes de décision du type :

$$
\text { Existe-t-il } x \in\{0,1\}^{n} \text { tel que }\left\{\begin{array}{cc}
c . x \geq z_{\min } & \text { et } \\
A . x \leq b & \text { et } \\
\sigma(x)=k &
\end{array}\right.
$$

où $\left(z_{\min }\right)$ est une suite positive strictement croissante. Il explore les zones de l'espace de recherche $\mathcal{X}_{k}$ pour lesquelles $z>z_{\text {min }}$. Son objectif premier n'est pas de maximiser un critère du type profit/ressource mais de minimiser $v_{b}$.

Nous notons $\mid$ R.L.| la taille de la running list qui correspond au nombre maximum d'itérations sans production d'une configuration $x$ réalisable $(A . x \leq b)$. Passé cette limite le processus s'interrompt. S'il ne trouve pas de configuration respectant les contraintes de sac, cet algorithme retourne le vecteur nul. Enfin pour des raisons de clarté le facteur aléatoire a été omis. Sa mise en œuvre consiste simplement à parcourir les composantes du vecteur $x$ dans un ordre aléatoire.

Une analyse de la complexité de $R L^{\text {tabou }}$ sera faite en section 7 à la suite de la présentation des résultats.

\footnotetext{
${ }^{7}$ L'objet ici est de trouver une solution mais pas de prouver qu'il n'en existe pas.
} 


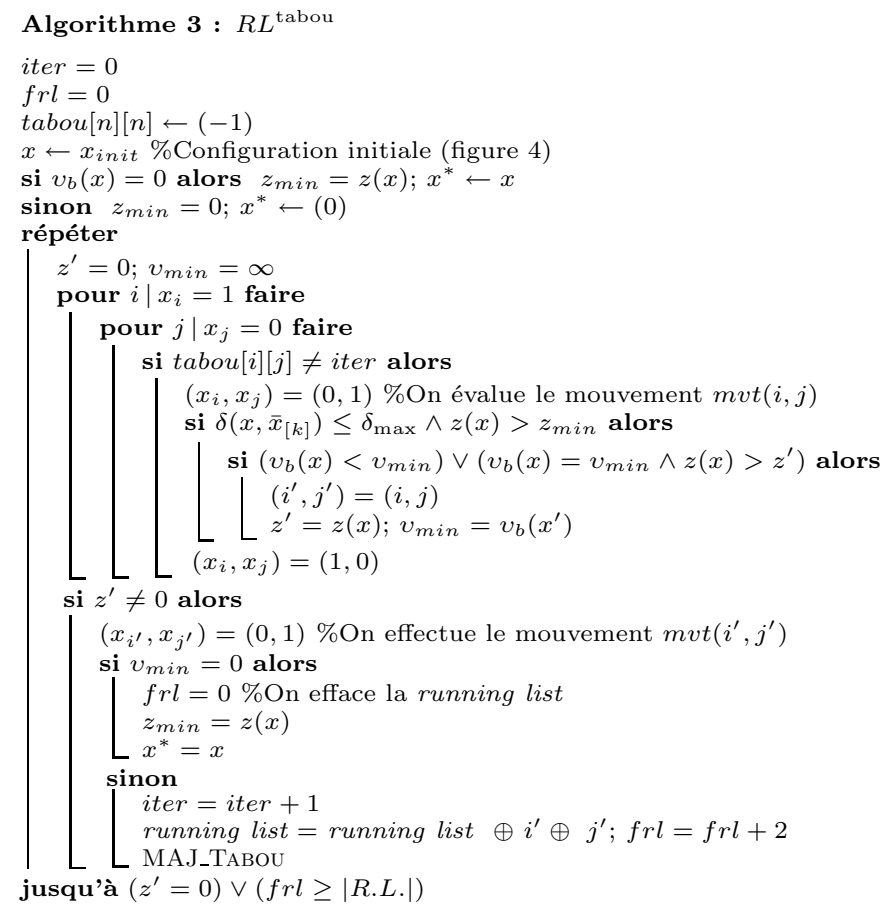

\section{RÉSUltats}

Nous avons expérimenté notre approche sur 3 séries de jeux tests. Pour toutes ces instances $R L^{\text {tabou }}$ a été exécuté, de façon distribuée, avec les 10 germes $(0 . .9)$ de la fonction standard srand(). L'algorithme est codé en $\mathrm{C}$ et tourne sur des configurations (machine/système) aussi diverses que: (PII350/Win.NT), (PII450/Win.NT), (PIII500/Win.NT), (Ultra Sparc 5/Unix) et (Ultra Sparc 30/Unix). Les temps d'exécution en secondes, qui figurent dans les colonnes t.t., sec.* et sec., sont donc donnés seulement à titre indicatif.

\subsection{JEUX CLASSIQUES}

Nous commençons par les 56 instances classiques que l'on retrouve notamment dans $[1,2,5,7,8,10-12,20,24,31,32]$. Ces jeux ne sont plus considérés comme difficiles aujourd'hui $[7,23]$. Cependant :

- d'une part ils constituent une référence à laquelle une méthode approchée ne peut se soustraire sans arguments a priori;

- d'autre part ils permettront une analyse comparative des valeurs $\delta_{[k]}$ qui fournira, justement, un début d'argumentation pour caractériser a priori la difficulté des problèmes. 
Nous avons, pour ces jeux, fixé la taille de la running list à $2000(\mid$ R.L. $\mid=2000)$. Les résultats sont présentés dans les tableaux 1 et 2 .

TABLEAU 1. Instances classiques.

\begin{tabular}{|c|c|c|r|r|r|r|r|r|}
\hline Pb. & $n \times m$ & {$[k]$} & $z^{*}$ & $k^{*}$ & $\delta^{*}$ & $\delta_{\left[k^{*}\right]}$ & iter $^{*}$ & t.t. \\
\hline \hline fp1 & $27 \times 4$ & $14 . .23$ & 3090 & 17 & 4.14 & 6.00 & 79 & 0 \\
\hline fp2 & $34 \times 4$ & $14 . .28$ & 3186 & 23 & 7.48 & 6.00 & 7 & 1 \\
\hline fp3 & $19 \times 2$ & $2 . .6$ & 28642 & 3 & 2.73 & 2.00 & 1 & 0 \\
\hline fp4 & $29 \times 2$ & $11 . .20$ & 95168 & 14 & 2.18 & 2.00 & 1 & 1 \\
\hline fp5 & $20 \times 10$ & $9 . .11$ & 2139 & 10 & 3.55 & 8.00 & 1 & 0 \\
\hline fp6 & $40 \times 30$ & $7 . .12$ & 776 & 9 & 5.16 & 8.00 & 27 & 1 \\
\hline fp7 & $37 \times 30$ & $15 . .20$ & 1035 & 17 & 3.55 & 8.00 & 1 & 1 \\
\hline peter1 & $6 \times 10$ & $3 . .4$ & 3800 & 3 & 1.16 & 4.00 & 0 & 0 \\
\hline peter2 & $10 \times 10$ & $3 . .7$ & 87061 & 5 & 4.25 & 2.00 & 2 & 0 \\
\hline peter3 & $15 \times 10$ & $8 . .11$ & 4015 & 9 & 1.38 & 2.00 & 1 & 0 \\
\hline peter4 & $20 \times 10$ & $9 . .11$ & 6120 & 9 & 0.07 & 2.00 & 0 & 0 \\
\hline peter5 & $28 \times 10$ & $15 . .18$ & 12400 & 18 & 2.00 & 2.00 & 1 & 1 \\
\hline peter6 & $39 \times 5$ & $24 . .33$ & 10618 & 27 & 4.14 & 6.00 & 331 & 9 \\
\hline peter7 & $50 \times 5$ & $26 . .40$ & 16537 & 35 & 7.34 & 8.00 & 852 & 31 \\
\hline hp1 & $28 \times 4$ & $15 . .24$ & 3418 & 18 & 4.14 & 6.00 & 79 & 3 \\
\hline hp2 & $35 \times 4$ & $14 . .28$ & 3186 & 23 & 7.34 & 8.00 & 7 & 9 \\
\hline weing1 & $28 \times 2$ & $12 . .15$ & 141278 & 14 & 2.00 & 2.00 & 1 & 1 \\
\hline weing2 & $28 \times 2$ & $10 . .12$ & 130883 & 11 & 2.00 & 4.00 & 1 & 1 \\
\hline weing3 & $28 \times 2$ & $5 . .9$ & 95677 & 6 & 2.73 & 2.00 & 1 & 2 \\
\hline weing4 & $28 \times 2$ & 10.17 & 119337 & 15 & 4.78 & 2.00 & 127 & 4 \\
\hline weing5 & $28 \times 2$ & $6 . .9$ & 98796 & 9 & 0.09 & 2.00 & 0 & 0 \\
\hline weing6 & $28 \times 2$ & $10 . .12$ & 130623 & 11 & 4.00 & 2.00 & 19 & 0 \\
\hline weing7 & $105 \times 2$ & $86 . .90$ & 1095445 & 87 & 2.62 & 4.00 & 1 & 8 \\
\hline weing8 & $105 \times 2$ & $27 . .36$ & 624319 & 30 & 2.18 & 2.00 & 1 & 24 \\
\hline sento1 & $60 \times 30$ & $18 . .23$ & 7772 & 20 & 5.16 & 8.00 & 27 & 16 \\
\hline sento2 & $60 \times 30$ & $31 . .36$ & 8722 & 33 & 3.55 & 8.00 & 1 & 10 \\
\hline
\end{tabular}

$P L / R L^{\text {tabou }}$ trouve la solution optimale (connue) dans tous les cas $\left(z^{*}=\dot{z}\right)$. Les valeurs de la phase $P L$ n'ont pas d'intérêt en tant que bornes puisque l'optimum est connu. Nous indiquons tout de même l'intervalle $[k]$ des hyperplans $\sigma(x)=k$ explorés par $R L^{\text {tabou}}$. Pour cette série et la suivante (à des fins de comparaison) nous donnons la distance $\delta^{*}$ de la meilleure solution $x^{*}$ à $\bar{x}_{\left[k^{*}\right]}$, ainsi que la valeur $\delta_{\left[k^{*}\right]}$ (Sect. 5.2) à partir de laquelle on estime le rayon de recherche autour du point $\bar{x}_{\left[k^{*}\right]}$. La colonne iter $^{*}$ correspond au nombre de mouvements pour atteindre $x^{*}$. La colonne t.t. représente la somme des temps sur tous les processus dans chaque hyperplan $\sigma(x)=k$. 
TABleau 2. Instances classiques (suite et fin).

\begin{tabular}{|c|c|c|r|r|r|r|r|r|}
\hline Pb. & $n \times m$ & {$[k]$} & $z^{*}$ & $k^{*}$ & $\delta^{*}$ & $\delta_{\left[k^{*}\right]}$ & iter $^{*}$ & $t . t$. \\
\hline \hline weish01 & $30 \times 5$ & $12 . .14$ & 4554 & 12 & 0.72 & 2.00 & 0 & 2 \\
\hline weish02 & $30 \times 5$ & $13 . .15$ & 4536 & 14 & 3.28 & 2.00 & 3 & 2 \\
\hline weish03 & $30 \times 5$ & $12 . .14$ & 4115 & 12 & 1.41 & 2.00 & 1 & 2 \\
\hline weish04 & $30 \times 5$ & $11 . .12$ & 4561 & 12 & 2.00 & 2.00 & 1 & 1 \\
\hline weish05 & $30 \times 5$ & $11 . .12$ & 4514 & 12 & 2.00 & 2.00 & 1 & 1 \\
\hline weish06 & $40 \times 5$ & $20 . .21$ & 5557 & 20 & 3.20 & 2.00 & 8 & 1 \\
\hline weish07 & $40 \times 5$ & $19 . .20$ & 5567 & 20 & 2.70 & 2.00 & 1 & 2 \\
\hline weish08 & $40 \times 5$ & $21 . .22$ & 5605 & 21 & 2.55 & 2.00 & 1 & 2 \\
\hline weish09 & $40 \times 5$ & $15 . .16$ & 5246 & 16 & 0.19 & 2.00 & 0 & 1 \\
\hline weish10 & $50 \times 5$ & $19 . .23$ & 6339 & 21 & 2.69 & 2.00 & 1 & 5 \\
\hline weish11 & $50 \times 5$ & $18 . .20$ & 5643 & 19 & 0.82 & 4.00 & 0 & 4 \\
\hline weish12 & $50 \times 5$ & $20 . .22$ & 6339 & 21 & 2.48 & 2.00 & 1 & 3 \\
\hline weish13 & $50 \times 5$ & $19 . .22$ & 6159 & 21 & 2.30 & 2.00 & 1 & 4 \\
\hline weish14 & $60 \times 5$ & $23 . .26$ & 6954 & 26 & 0.63 & 4.00 & 0 & 5 \\
\hline weish15 & $60 \times 5$ & $26 . .27$ & 7486 & 26 & 2.00 & 4.00 & 1 & 3 \\
\hline weish16 & $60 \times 5$ & $25 . .28$ & 7289 & 26 & 2.44 & 2.00 & 1 & 4 \\
\hline weish17 & $60 \times 5$ & $38 . .41$ & 8633 & 41 & 2.00 & 2.00 & 1 & 4 \\
\hline weish18 & $70 \times 5$ & $39 . .41$ & 9580 & 40 & 2.26 & 2.00 & 1 & 4 \\
\hline weish19 & $70 \times 5$ & $26 . .29$ & 7698 & 27 & 2.00 & 2.00 & 1 & 7 \\
\hline weish20 & $70 \times 5$ & $35 . .37$ & 9450 & 35 & 0.31 & 2.00 & 0 & 4 \\
\hline weish21 & $70 \times 5$ & $32 . .35$ & 9074 & 32 & 2.00 & 2.00 & 1 & 6 \\
\hline weish22 & $80 \times 5$ & $32 . .35$ & 8947 & 33 & 4.00 & 2.00 & 12 & 7 \\
\hline weish23 & $80 \times 5$ & $30 . .33$ & 8344 & 32 & 4.00 & 2.00 & 461 & 12 \\
\hline weish24 & $80 \times 5$ & $45 . .47$ & 10220 & 45 & 0.06 & 2.00 & 0 & 8 \\
\hline weish25 & $80 \times 5$ & $40 . .42$ & 9939 & 40 & 2.00 & 2.00 & 1 & 7 \\
\hline weish26 & $90 \times 5$ & $35 . .39$ & 9584 & 36 & 2.00 & 2.00 & 1 & 14 \\
\hline weish27 & $90 \times 5$ & $37 . .39$ & 9819 & 38 & 1.43 & 2.00 & 1 & 7 \\
\hline weish28 & $90 \times 5$ & $36 . .38$ & 9492 & 37 & 1.34 & 4.00 & 1 & 9 \\
\hline weish29 & $90 \times 5$ & $36 . .38$ & 9410 & 36 & 0.02 & 2.00 & 0 & 6 \\
\hline weish30 & $90 \times 5$ & $50 . .51$ & 11191 & 51 & 2.00 & 2.00 & 1 & 2 \\
\hline
\end{tabular}

Nous ne faisons pas figurer le nombre total d'itérations effectuées par $R L^{\text {tabou }}$ puisqu'il peut se déduire par la simple formule :

$$
\text { iter }^{*}+\left(\frac{1}{2} \times \mid \text { R.L. } \mid\right) .
$$

Nous remarquons qu'en général $\delta^{*}$, qui est pour ces jeux la distance de la solution optimale à $\bar{x}_{[k]}$, est assez faible. Cela explique la rapidité de notre algorithme à résoudre ces instances. On constate par ailleurs que sur 10 instances $\left(\right.$ iter $\left.^{*}=0\right)$, l'heuristique du plus proche point suffit à construire la solution optimale et qu'il suffit d'un mouvement pour 31 autres instances. Pour ces 41 instances il n'est pas utile de mettre en œuvre la métaheuristique tabou pour trouver l'optimum. 
Nous rappelons que t.t. est le temps total, pour 10 relances de 1000 itérations (une par germe aléatoire), pour chaque hyperplan : ainsi t.t. est-il plus grand pour weish 26 que pour weish 23 . weish 26 contient 10 variables de plus et exige l'exploration d' un hyperplan supplémentaire. iter* est le nombre d'itérations, dans l'hyperplan $\sigma(x)=k^{*}$, pour atteindre la meilleure configuration : sa valeur n'est pas obligatoirement corrélée à celle de t.t.

\subsection{Jeux Glover et Kochenberger}

Cette deuxième série de tests est constituée des 7 dernières instances proposées par Glover et Kochenberger [20]. Le tableau 3 résume les valeurs clés de la phase $P L . \quad \overline{\bar{z}}=\max \left(\bar{z}_{[k]}\right)$ est la meilleure valeur parmi les optima des programmes $\mathrm{MKP}_{[k]}$. La dernière colonne (sec.) indique les durées cumulées, en secondes, pour la résolution de MKP ainsi que des $\mathrm{MKP}_{[k]}$.

TABleau 3. Phase $P L$ sur GK18 $\leftrightarrow$ GK24.

\begin{tabular}{|c|c|c|c|c|c|}
\hline GK & $n \times m$ & {$[k]$} & $\overline{\bar{z}}$ & $\bar{z}$ & sec. \\
\hline \hline 18 & $100 \times 25$ & $58 . .64$ & 4545.66 & 4545.79 & 4 \\
\hline 19 & $100 \times 25$ & $49 . .55$ & 3886.45 & 3886.80 & 4 \\
\hline 20 & $100 \times 25$ & $61 . .74$ & 5198.54 & 5198.64 & 5 \\
\hline 21 & $100 \times 25$ & $40 . .46$ & 3219.73 & 3219.92 & 5 \\
\hline 22 & $100 \times 25$ & $31 . .37$ & 2544.02 & 2544.03 & 4 \\
\hline 23 & $200 \times 15$ & $119 . .126$ & 9245.53 & 9245.67 & 12 \\
\hline 24 & $500 \times 25$ & $116 . .125$ & 9080.44 & 9080.45 & 220 \\
\hline
\end{tabular}

TABleau 4. Phase $R L^{\text {tabou }}$ sur GK18 GK24.

\begin{tabular}{|c||c|c||c|c|c|c|c|c|c|}
\hline GK & $T_{H F}$ & $T_{H F}^{\prime}$ & $z^{*}$ & $\delta^{*}$ & $\delta_{\left[k^{*}\right]}$ & $k^{*}$ & iter $^{*}$ & sec. $^{*}$ & sec. \\
\hline \hline 18 & 4524 & 4526 & $\mathbf{4 5 2 8}$ & 13.55 & 24.00 & 61 & 3683 & 10 & 395 \\
\hline 19 & 3866 & 3867 & $\mathbf{3 8 6 9}$ & 15.29 & 20.00 & 51 & 3144 & 9 & 382 \\
\hline 20 & 5177 & 5179 & $\mathbf{5 1 8 0}$ & 15.95 & 22.00 & 70 & 2080 & 5 & 366 \\
\hline 21 & 3195 & 3197 & $\mathbf{3 2 0 0}$ & 10.78 & 22.00 & 42 & 1465 & 4 & 366 \\
\hline 22 & 2521 & $\mathbf{2 5 2 3}$ & $\mathbf{2 5 2 3}$ & 14.24 & 28.00 & 34 & 512 & 2 & 383 \\
\hline 23 & 9231 & 9233 & $\mathbf{9 2 3 5}$ & 19.69 & 16.00 & 123 & 16976 & 131 & 723 \\
\hline 24 & 9062 & 9064 & $\mathbf{9 0 7 0}$ & 15.21 & 22.00 & 119 & 9210 & 268 & 2027 \\
\hline
\end{tabular}

Le tableau 4 correspond à la phase recherche locale. Pour cette série de problèmes, ainsi que la suivante, la taille de la running list est fixée à 100000 . La colonne $T_{H F}$ indique les résultats obtenus par l'algorithme tabou de Hanafi et Fréville [23] que l'on peut comparer avec la valeur $z^{*}$, signalée en caractères gras, de la meilleure configuration $x^{*}$, à $k^{*}$ objets, trouvée par $R L^{\text {tabou}}$. La colonne $T_{H F}^{\prime}$ contient les récentes valeurs obtenues par Hanafi avec son algorithme tabou. Les colonnes sec.* et sec. indiquent respectivement le temps d'obtention de $x^{*}$ et 
le temps total d'exécution d'une phase $R L^{\text {tabou }}$. Nous améliorons strictement la majorité des résultats sur ces instances.

Par rapport aux jeux précédents (Sect. 6.1), le nombre d'itérations et, par voie de conséquence le temps CPU, nécessaires à l'obtention de ces solutions ont augmentés. En effet :

- les nombres $n$ et $m$, plus grands pour ces instances, interviennent dans la complexité de la fonction d'évaluation du voisinage ;

- $\delta_{\left[k^{*}\right]}$ a augmenté et $C_{n}^{\delta_{\left[k^{*}\right]} 8}$ donne une idée de la combinatoire à traiter.

Notre processus doit parcourir un espace de recherche $\mathcal{X}_{k}$ plus vaste. À échantillonnage équivalent de $\mathcal{X}_{k}$, il faut plus d'itérations. Comme nous l'avons évoqué au début de la section sur les jeux classiques nous avons, avec les valeurs $\delta_{[k]}$, une mesure expérimentale de la difficulté d'une instance de MKP01.

\subsection{Jeux Chu et Beasley}

270 instances de MKP01 de tailles allant de 100 à 500 variables et de 5 à 30 contraintes sont proposées, très récemment, par Chu et Beasley [7] et constituent une partie de la OR-LiBRARY ${ }^{9}$.

TABLEAU 5. 24 instances CBm.n.r.

\begin{tabular}{|r|c|c||c||c|c|c|c|}
\hline CBm.n.r & {$[k]$} & $\bar{z}$ & $A G_{C B}$ & $z^{*}$ & $k^{*}$ & iter $^{*}$ & sec. $^{*}$ \\
\hline \hline 5.100 .0 & $28 . .31$ & 24585.90 & 24381 & 24381 & 29 & 1 & 1 \\
\hline 5.100 .10 & $51 . .55$ & 42939.52 & 42757 & 42757 & 52 & 187 & 1 \\
\hline 5.100 .20 & $75 . .78$ & 60016.56 & 59822 & 59822 & 78 & 0 & 0 \\
\hline 10.100 .0 & $26 . .29$ & 23480.64 & 23064 & 23064 & 27 & 2749 & 3 \\
\hline 10.100 .10 & $50 . .53$ & 41712.64 & 41395 & 41395 & 51 & 173 & 1 \\
\hline 10.100 .20 & $75 . .78$ & 57626.33 & 57375 & 57375 & 77 & 1 & 1 \\
\hline 30.100 .0 & $23 . .26$ & 22579.07 & 21946 & 21946 & 24 & 420 & 1 \\
\hline 30.100 .10 & $48 . .51$ & 41276.36 & 40767 & 40767 & 49 & 7625 & 15 \\
\hline 30.100 .20 & $73 . .75$ & 57987.77 & 57494 & 57494 & 73 & 42 & 1 \\
\hline 5.250 .0 & $71 . .74$ & 59442.47 & 59312 & 59312 & 73 & 252 & 1 \\
\hline 5.250 .10 & $130 . .135$ & 109220.64 & 109109 & 109109 & 132 & 2984 & 10 \\
\hline 5.250 .20 & $189 . .194$ & 149765.67 & 149659 & 149659 & 192 & 9663 & 31 \\
\hline 10.250 .0 & $66 . .70$ & 59489.34 & 59187 & 59187 & 68 & 12373 & 60 \\
\hline 10.250 .10 & $126 . .131$ & 111147.15 & 110863 & $\mathbf{1 1 0 8 8 9}$ & 128 & 468 & 2 \\
\hline 10.250 .20 & $186 . .190$ & 152031.39 & 151790 & $\mathbf{1 5 1 8 0 1}$ & 188 & 24536 & 99 \\
\hline 30.250 .0 & $61 . .65$ & 57430.15 & 56693 & $\mathbf{5 6 7 9 6}$ & 63 & 2221 & 14 \\
\hline 30.250 .10 & $123 . .128$ & 108258.07 & 107689 & $\mathbf{1 0 7 7 7 0}$ & 125 & 65392 & 1399 \\
\hline 30.250 .20 & $186 . .190$ & 150574.32 & 150083 & $\mathbf{1 5 0 1 6 3}$ & 187 & 27015 & 332 \\
\hline 5.500 .0 & $144 . .149$ & 120234.92 & 120130 & $\mathbf{1 2 0 1 3 4}$ & 146 & 39350 & 656 \\
\hline 5.500 .10 & $265 . .270$ & 218500.08 & 218422 & $\mathbf{2 1 8 4 2 6}$ & 267 & 6761 & 90 \\
\hline 5.500 .20 & $381 . .387$ & 295896.38 & 295828 & 295828 & 383 & 5012 & 72 \\
\hline 10.500 .0 & $132 . .139$ & 118019.48 & 117726 & $\mathbf{1 1 7 7 4 6}$ & 134 & 55794 & 776 \\
\hline 10.500 .10 & $254 . .260$ & 217552.92 & 217318 & $\mathbf{2 1 7 3 4 3}$ & 256 & 48536 & 648 \\
\hline 10.500 .20 & $376 . .382$ & 304555.03 & 304344 & $\mathbf{3 0 4 3 5 0}$ & 379 & 68008 & 1321 \\
\hline
\end{tabular}

\footnotetext{
${ }^{8}$ Nous rappelons que $\delta_{\left[k^{*}\right]}$ est un entier pair (Sect. 5.2).

9 accessible à l'adresse : http//mscmga.ms.ic.ac.uk/.
} 
Nous présentons, dans un premier temps, un tableau synthétique sur 24 instances couvrant toutes les caractéristiques offertes par leurs auteurs $\left(n \times m \times \alpha^{10}\right)$ exceptées $n=500$ et $m=30$ qui font l'objet d'un autre tableau. Le format générique du libellé de ces instances est le suivant : CBm.n. $r$ avec $0 \leq r \leq 29$. La colonne $n \times m$ n'est donc plus utile. De même, la valeur $\alpha$ peut se déduire à partir du rang $r$ de l'instance : $0 \leq r \leq 9 \Rightarrow \alpha=0.25,10 \leq r \leq 19 \Rightarrow \alpha=0.50$ et $20 \leq r \leq 29 \Rightarrow \alpha=0.75$. De façon similaire à la section précédente, la colonne $A G_{C B}$ représente les meilleures valeurs obtenues par l'algorithme génétique de Chu et Beasley [7]. Les valeurs optimales, lorsqu'elles sont connues, figurent en italique. $R L^{\text {tabou }}$ produit bien une solution optimale dans ce cas. Nous améliorons la majorité des résultats pour lesquels cet optimum n'est pas connu (valeurs indiquées en caractères gras).

Nous améliorons également de manière significative les meilleurs résultats obtenus par Chu et Beasley [7] sur les 30 instances les plus importantes de la ORLiBrARY ( $c f$. Tab. 6). Sur cette série d'instances, la valeur $\overline{\bar{z}}$ est sensiblement plus fine que la borne $\bar{z}$.

TABleau 6. 30 instances CB30.500.

\begin{tabular}{|c|c|c|c|c|c|c|c|c|}
\hline$r$ & {$[k]$} & $\overline{\bar{z}}$ & $\bar{z}$ & $A G_{C B}$ & $z^{*}$ & $k^{*}$ & iter $^{*}$ & sec. $^{*}$ \\
\hline$\overline{00}$ & $\overline{128 . .133}$ & 1116601.41 & 1116619.01 & 1115868 & 1115950 & 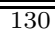 & $\overline{17841}$ & 397 \\
\hline 1 & $125 . .131$ & 115365.72 & 115370.13 & 114667 & 114810 & 128 & 104866 & 2264 \\
\hline 2 & $125 . .132$ & 117330.33 & 117342.45 & 116661 & 116683 & 128 & 73590 & 1203 \\
\hline 3 & $125 . .131$ & 115936.14 & 115946.40 & 115237 & 115301 & 128 & 71820 & 1587 \\
\hline 4 & $125 . .133$ & 117078.97 & 117079.29 & 116353 & 116435 & 127 & 75909 & 1784 \\
\hline 5 & $128 . .134$ & 116362.03 & 116377.55 & 115604 & 115694 & 131 & 33391 & 684 \\
\hline 6 & $126 . .132$ & 114682.47 & 114689.65 & 113952 & 114003 & 128 & 107994 & 2851 \\
\hline 7 & $125 \ldots 132$ & 114833.73 & 114847.83 & 114199 & 114213 & 129 & 87593 & 1503 \\
\hline 8 & $125 . .132$ & 115901.29 & 115902.61 & 115247 & 115288 & 127 & 75243 & 1495 \\
\hline 9 & $125 . .132$ & 117661.71 & 117668.77 & 116947 & 117055 & 129 & 39044 & 869 \\
\hline 10 & $249 . .254$ & 218597.06 & 218601.52 & 217 & 218068 & 251 & 6828 & 116 \\
\hline 11 & $249 . .254$ & 215074.67 & 215074.71 & 214534 & 214562 & 251 & 89201 & 2478 \\
\hline 12 & $248 . .253$ & 216395.94 & 216401.07 & 215854 & 215903 & 250 & 60074 & 1311 \\
\hline 13 & $249 . .255$ & 218345.99 & 218350.48 & 217836 & 217910 & 251 & 50732 & 1121 \\
\hline 14 & $248 . .254$ & 216094.49 & 216094.51 & 215566 & 215596 & 251 & 62524 & 1262 \\
\hline 15 & $250 . .257$ & 216326.72 & 216327.35 & 215762 & 215842 & 253 & 34201 & 633 \\
\hline 16 & $250 . .256$ & 216375.27 & 216376.30 & 215772 & 215838 & 252 & 54476 & 1003 \\
\hline 17 & $250 . .257$ & 217013.40 & 217014.09 & 216336 & 216419 & 253 & 40683 & 947 \\
\hline 18 & $250 . .257$ & 217830.74 & 217839.18 & 217290 & 217305 & 253 & 64489 & 1475 \\
\hline 19 & $250 . .256$ & 215218.07 & 215218.48 & 214624 & 214671 & 252 & 18531 & 368 \\
\hline 20 & $373 . .378$ & 302038.59 & 3020 & 301627 & 301643 & 375 & 1298 & 17 \\
\hline 21 & $372 . .379$ & 300453.13 & 300455.00 & 299985 & 300055 & 374 & 78278 & 1532 \\
\hline 22 & $373 . .379$ & 305499.91 & 305501.21 & 304995 & 305028 & 375 & 64926 & 1161 \\
\hline 23 & $373 . .379$ & 302447.30 & 302456.21 & 301935 & 302004 & 375 & 26901 & 1110 \\
\hline 24 & $374 \ldots 379$ & 304895.74 & 304901.35 & 304404 & 304411 & 376 & 20483 & 333 \\
\hline 25 & $371 . .377$ & 297409.08 & 297409.44 & 296894 & 296961 & 374 & 31403 & 462 \\
\hline 26 & $372 . .377$ & 303763.56 & 303765.88 & 303233 & 303328 & 373 & 43398 & 757 \\
\hline 27 & $374 . .380$ & 307397.96 & 307402.50 & 306944 & 306999 & 376 & 33810 & 1366 \\
\hline 28 & $373 . .379$ & 303605.11 & 303605.92 & 303057 & 303080 & 374 & 17647 & 350 \\
\hline 29 & $373 . .379$ & 301014.80 & 301020.63 & 300460 & 300532 & 376 & 6948 & 150 \\
\hline
\end{tabular}

\footnotetext{
${ }^{10} \alpha$ est le taux de débordement des ressources : $b_{i} / \sum_{j=1}^{n} A_{i j}$.
} 
La taille de la running list étant fixée à 100000 , nous savons que l'algorithme a effectué 50000 itérations après iter* . Nous donnons, dans la section suivante, une analyse de la complexité temporelle de cette fin de processus d'optimisation.

Nous terminons cette étude comparative par la synthèse des résultats sur l'ensemble des jeux à 500 variables de la OR-LiBrary. Nous ajoutons les résultats obtenus par Osorio et al. [29] lors de récents travaux dont l'objectif était d'améliorer les performances de l'outil de programmation linéaire en nombres entiers CPLEX (V6.5.2) ; travaux qui portent sur un algorithme de fixation de variables avec adjonction de coupes (résultats en colonne Fix+Cuts). Nous retranscrivons également, en colonne CPLEX, les valeurs trouvées par ce logiciel sans les modifications effectuées par les auteurs du rapport [29].

TABleau 7. Moyennes des meilleurs résultats par groupe de 10 instances $\mathrm{CB} m .500$.

\begin{tabular}{|c|c|c|c|c|c|c|}
\hline$m$ & $\alpha$ & $A G_{C B}$ & Fix+Cuts & $C P L E X$ & $P L / R L$ & $\left(\bar{z}-z^{*}\right) / \bar{z}$ \\
\hline 5 & $1 / 4$ & 120616 & 120610 & 120619 & $\mathbf{1 2 0 6 2 3}$ & 0.0008 \\
& $1 / 2$ & 219503 & 219504 & 219506 & $\mathbf{2 1 9 5 0 7}$ & 0.0004 \\
& $3 / 4$ & 302355 & $\mathbf{3 0 2 3 6 1}$ & 302358 & 302360 & 0.0002 \\
\hline \multirow{2}{*}{10} & $1 / 4$ & 118566 & 118584 & 118597 & $\mathbf{1 1 8 6 0 0}$ & 0.0020 \\
& $1 / 2$ & 217275 & 217297 & 217290 & $\mathbf{2 1 7 2 9 8}$ & 0.0009 \\
& $3 / 4$ & 302556 & 302562 & 302573 & $\mathbf{3 0 2 5 7 5}$ & 0.0007 \\
\hline 30 & $1 / 4$ & 115470 & 115520 & 115497 & $\mathbf{1 1 5 5 4 7}$ & 0.0055 \\
& $1 / 2$ & 216187 & 216180 & 216151 & $\mathbf{2 1 6 2 1 1}$ & 0.0024 \\
& $3 / 4$ & 302353 & 302373 & 302366 & $\mathbf{3 0 2 4 0 4}$ & 0.0015 \\
\hline
\end{tabular}

Fix + Cuts et CPLEX sont interrompus après 3 heures de calcul (sur un PIII500) ou lorsque l'arbre de recherche dépasse les 250 Mo d'occupation mémoire. Nous constatons que, plus le nombre de contraintes est élevé, plus la moyenne des écarts à l'optimum continu est grande (dernière colonne), plus notre approche se distingue des autres algorithmes en conservant une avance qualitative significative.

\section{Analyse De la COMPlexité}

\subsection{VOISINAGE ET running list}

L'estimation du temps CPU pris par $R L^{\text {tabou }}$ en fonction du nombre total d'itérations n'est pas facile. En effet la running list est remise à zéro à chaque fois que l'on rencontre une configuration réalisable (Sect. 5.4). Étant donné que toute phase $R L^{\text {tabou }}$ finit par un nombre constant $(|R . L| / 2$.$) de mouvements on peut$ proposer, pour cette étape, la formule suivante :

$$
\text { sec. } \approx(\mathcal{A} \times \text { iter } \times(n-k) \times k \times m)+\left(\mathcal{B} \times \frac{\text { iter } \times(\text { iter }-1)}{2}\right)
$$


où : $\mathcal{A}$ représente le coût moyen, en secondes par itération, du calcul de la fonction d'évaluation du voisinage, $\mathcal{B}$ celui de la mise à jour de la liste tabou. Dans cette formule, le coefficient $\mathcal{A}$ est assez imprécis : en effet nous nous limitons à un rayon $\delta_{\max }$ autour d'un point $\bar{x}_{[k]}$. Nous n'avons donc pas systématiquement la mesure de $z(x)$, mais surtout celle de $v_{b}(x)$ qui coûte $m$, à effectuer. Faute de pouvoir évaluer correctement $\mathcal{A}$ et $\mathcal{B}$ nous avons regroupé dans le tableau 8

Tableau 8. Temps de calcul en secondes.

\begin{tabular}{|c|c|c|c|c|c|c|c|c|c|}
\hline $\mid$ R.L. $\mid$ & $\begin{array}{c}n=100 \\
m=5\end{array}$ & $\begin{array}{c}100 \\
10\end{array}$ & $\begin{array}{c}100 \\
30\end{array}$ & $\begin{array}{c}250 \\
5\end{array}$ & $\begin{array}{c}250 \\
10\end{array}$ & $\begin{array}{c}250 \\
30\end{array}$ & $\begin{array}{c}500 \\
5\end{array}$ & $\begin{array}{c}500 \\
10\end{array}$ & $\begin{array}{c}500 \\
30\end{array}$ \\
\hline \hline 25000 & 21 & 22 & 25 & 56 & 59 & 82 & 199 & 222 & 244 \\
\hline 50000 & 70 & 73 & 78 & 142 & 148 & 193 & 425 & 479 & 526 \\
\hline 100000 & 253 & 262 & 273 & 410 & 424 & 512 & 1001 & 1106 & 1193 \\
\hline 200000 & 1046 & 1076 & 1100 & 1371 & 1427 & 1608 & 2566 & 2771 & 2975 \\
\hline
\end{tabular}

les temps d'exécution sur des instances à $\alpha=0.50$ pour toutes les combinaisons $n \times m$ et pour 4 tailles de la running list. La machine utilisée est un PIII500. Nous avons modifié l'algorithme $R L^{\text {tabou }}$ en supprimant la remise à zéro de la running list pour avoir un nombre d'itérations directement lié à la taille de cette dernière. Le facteur quadratique dû à la gestion de la liste tabou est assez faible. Le temps maximum est de moins de 20 minutes pour $\mid$ R.L. $\mid=100000$. C'est la valeur à rajouter aux temps indiqués dans les colonnes $s e c .^{*}$ des tableaux 5 et 6 .

Dans le cas d'une parallélisation des processus tabou avec une machine par germe de $\operatorname{srand}()$, ce temps n'est pas prohibitif. Nous pouvons même accroître la taille de la running list pour tenter d'améliorer encore les résultats (Tab. 9) : nous

Tableau 9. Augmentation de la running list.

\begin{tabular}{|c|c|c|c|c|c|c|c|}
\cline { 2 - 8 } \multicolumn{1}{c|}{} & \multicolumn{2}{c|}{$\mid$ R.L. $\mid=100000$} & \multicolumn{4}{c|}{$\mid$ R.L. $\mid=300000$} & \multicolumn{1}{c|}{} \\
\hline $\mathrm{CB}$ & $\delta_{1}^{*}$ & $z_{1}^{*}$ & $z_{2}^{*}$ & $\delta_{2}^{*}$ & iter $^{*}$ & t.t. & $\delta\left(x_{1}^{*}, x_{2}^{*}\right)$ \\
\hline \hline 5.500 .10 & 5.8 & 218426 & $\mathbf{2 1 8 4 2 8}$ & 9.26 & 173312 & 6441 & 14.0 \\
\hline 30.500 .0 & 15.93 & 115950 & $\mathbf{1 1 5 9 9 1}$ & 18.61 & 264408 & 9760 & 24.0 \\
\hline
\end{tabular}

constatons un gain par rapport aux valeurs des tableaux 5 et 6 . Nous remarquons aussi que les distances $\delta_{2}^{*}$ aux optima $\bar{x}_{[267]}$ et $\bar{x}_{[130]}$ ont augmenté. En terme de combinatoire la distance $\delta\left(x_{1}^{*}, x_{2}^{*}\right)$ entre la nouvelle solution $x_{2}^{*}$ et la précédente $x_{1}^{*}$ est relativement importante. Le processus tabou a exploré plus loin. Le temps total t.t. (en secondes) devient important mais cette évolution nous encourage à travailler sur la complexité de la procédure $R L^{\text {tabou }}$.

\subsection{LE PARAMÈTRE $\delta_{\max }$}

Enfin le paramètre $\delta_{\max }$ est un facteur crucial de l'efficacité de notre algorithme. Le tableau 10 illustre bien l'influence de $\delta_{\max }$ à la fois sur la stabilité de $R L^{\text {tabou }}$ 
vis-à-vis de la discrimination aléatoire des meilleurs candidats dans $\mathcal{N}(x)$ et sur le temps total d'exécution de $R L^{\text {tabou }}$. La colonne $g$ contient la valeur du germe de la fonction $\operatorname{srand}()$. Nous soulignons en caractère gras les temps minimums et maximums d'exécution complète de la phase tabou pour chacune des valeurs de $\delta_{\max }$.

TABLEAU 10. Influence de $\delta_{\max }$ sur $R L^{\text {tabou }}:$ GK2 $24, k^{*}=119$, $\delta_{[119]}=22$.

\begin{tabular}{|c|c|c|c|c|c||c|c|c|c|c|}
\hline \multirow{2}{*}{$g$} & \multicolumn{3}{|c||}{$\delta_{\max }=0.75 \times \delta_{[119]}=16.5$} & \multicolumn{4}{c|}{$\delta_{\max }=0.6 \times \delta[119]=13.2$} \\
\cline { 2 - 12 } & iter $^{*}$ & sec. $^{*}$ & sec. & $\delta^{*}$ & $z^{*}$ & iter $^{*}$ & sec. $^{*}$ & sec. & $\delta^{*}$ & $z^{*}$ \\
\hline 0 & 67354 & 2200 & 4014 & 10.87 & 9070 & 25172 & 626 & 2120 & 10.87 & 9070 \\
\hline 1 & 26735 & 763 & 2581 & 16.17 & 9067 & 68809 & 1924 & $\mathbf{3 4 0 9}$ & 10.87 & 9070 \\
\hline 2 & 46175 & 1373 & 3171 & 13.81 & 9067 & 30508 & 779 & 2297 & 10.87 & 9070 \\
\hline 3 & 15882 & 438 & 2282 & 14.75 & 9067 & 289 & 8 & $\mathbf{1 4 4 5}$ & 10.87 & 9070 \\
\hline 4 & 87218 & 2983 & $\mathbf{4 8 1 0}$ & 10.87 & 9070 & 1754 & 39 & 1470 & 10.87 & 9070 \\
\hline 5 & 4879 & 142 & 1949 & 16.04 & 9066 & 54029 & 1338 & 2809 & 10.87 & 9070 \\
\hline 6 & 79848 & 2509 & 4237 & 14.90 & 9068 & 71834 & 1838 & 3290 & 10.87 & 9070 \\
\hline 7 & 9210 & 268 & $\mathbf{2 0 2 7}$ & 15.21 & 9070 & 6758 & 150 & 1616 & 10.87 & 9070 \\
\hline 8 & 41101 & 1205 & 2901 & 14.69 & 9067 & 44282 & 1212 & 2633 & 10.87 & 9070 \\
\hline 9 & 17572 & 507 & 2234 & 10.87 & 9070 & 25378 & 624 & 2082 & 10.87 & 9070 \\
\hline
\end{tabular}

Remarquons toutefois que des valeurs $\delta_{\max }$ trop faibles risquent d'interdire des zones intéressantes de $\mathcal{X}_{k}\left(c f\right.$. valeurs de $\delta_{1}^{*}$ et $\delta_{2}^{*}$ Tab. 9). Ce paramètre de réglage augmente bien sûr la complexité globale de notre approche. Nous nous sommes limités à $\delta_{\max }=\operatorname{coe} f \times \delta$ avec coef $\in\{0.75,1,1.5,2\}$ et même exceptionnellement 3 pour certains jeux de la section 6.1 pour lesquels $\delta_{\left[k^{*}\right]}$ était trop petit (voir Tabs. 1 et 2).

\section{Conclusion}

Nous avons mis en œuvre une approche hybride très performante qui combine la programmation linéaire et la recherche locale tabou. Son principe général est d'utiliser la méthode du simplexe pour obtenir des points continus autour desquels lancer un algorithme tabou. Nous avons introduit une caractéristique intéressante $\left(\delta_{[k]}\right)$ pour les problèmes MKP01 et proposé une alternative au critère de choix profit/ressource pour le mécanisme de transformation locale d'une configuration $x$. Nous avons aussi développé une version relativement efficace de la méthode d'élimination inverse dont l'exploitation peut renforcer d'autres algorithmes tabou.

L'idée d'échantillonner $\{0,1\}^{n}$ autour d'optima de $[0-1]^{n}$ s'est avérée très bénéfique et performante. En effet, notre algorithme hybride améliore de manière significative les derniers résultats connus sur des instances difficiles [7,23]. 
Ce travail peut apporter une contribution dans le cadre des méthodes exactes et cela à deux titres :

- réduction de l'espace de recherche par encadrement du nombre de variables à l'optimum : la colonne $[k]$ des tableaux 3,5 et 6 donnent les valeurs potentiellement intéressantes pour l'optimum ;

- fixation de variables à partir d'un minorant $z$ de bonne qualité, sa configuration $x$ et les coûts réduits des variables hors base $[2,21]$ : si l'on applique ce principe sur l'instance GK024 à 500 variables, due à Glover et Kochenberger [20], $P L / R L^{\text {tabou }}$ nous fournit $x$, tel que $c . x=9070$ (Sect. 4). On peut alors fixer 38 variables à 0 .

Plusieurs voies d'amélioration sont encore à explorer :

- une étude plus précise sur la distance $\delta_{\max }$ qui limite l'espace de recherche autour des points continus $\bar{x}_{[k]}$ augmentera l'efficacité de $R L^{\text {tabou }}$;

- la mise en œuvre d'un mécanisme de relance permettra éventuellement de trouver des solutions de meilleure qualité ;

- la prise en compte, dans une version distribuée, d'une population d'optima locaux, pour intégrer de nouvelles contraintes aux programmes relaxés MKP $[k]$ puis, par le simplexe, générer de nouveaux points continus pour relancer la $R L$.

L'idée la plus intéressante semble bien être de transformer le schéma à sens unique $P L / R L^{\text {tabou}}$, qui correspond à une simple alternance entre le simplexe et tabou par une relation réactive $P L \leftrightarrow R L^{\text {tabou }}$ plus riche qui construirait, de manière dynamique, un problème relaxé dont la solution continue serait plus attractive pour la recherche locale.

Voici donc de nouvelles perspectives pour la résolution approchée du sac à dos multidimensionnel en variables bivalentes, qui nous l'espérons, apporteront des résultats encore meilleurs dans un proche avenir.

Remerciements. Nous tenons à remercier Saïd Hanafi pour nous avoir procuré les jeux de la série GK18 GK24 (Sect. 6.2) sur lesquels il a travaillé ainsi que pour ses amples commentaires sur la $M E I$.

\section{RÉFÉRENCES}

[1] R. Aboudi et K. Jörnsten, Tabu Search for General Zero-One Integer Programs using the Pivot and Complement Heuristic. ORSA J. Comput. 6 (1994) 82-93.

[2] E. Balas et C.H. Martin, Pivot and Complement a Heuristic for 0-1 Programming. Management Sci. 26 (1980) 86-96.

[3] R. Battiti et G. Tecchiolli, The reactive tabu search. ORSA J. Comput. 6 (1994) 128-140.

[4] R. Bellman et D. Stuart, Applied Dynamic Programming. Princeton University Press (1962).

[5] P. Boucher et G. Plateau, Étude des méthodes de bruitage appliquées au problème du sac à dos à plusieurs contraintes en variables $0-1$, dans $J N P C C^{\prime} 995^{\mathrm{es}}$ journées nationales sur la résolution pratique de problèmes NP-complets (1999) 151-162. 
[6] I. Charon et O. Hudry, The noising method: A new method for combinatorial optimization. Oper. Res. Lett. 14 (1993) 133-137.

[7] P.C. Chu et J.E. Beasley, A genetic algorithm for the multidimensional knapsack problem. J. Heuristic 4 (1998) 63-86.

[8] F. Dammeyer et S. Voß, Dynamic tabu list management using the reverse elimination method. Ann. Oper. Res. 41 (1993) 31-46.

[9] G.B. Dantzig, Discrete-variable extremum problems. Oper. Res. 5 (1957) 266-277.

[10] A. Drexl, A simulated annealing approach to the multiconstraint zero-one knapsack problem. Computing 40 (1988) 1-8.

[11] A. Fréville et G. Plateau, Heuristic and reduction methods for multiple constraints 0-1 linear programming problems. Eur. J. Oper. Res. 24 (1986) 206-215.

[12] A. Fréville et G. Plateau, Sac à dos multidimensionnel en variable 0-1 : encadrement de la somme des variables à l'optimum. RAIRO: Oper. Res. 27 (1993) 169-187.

[13] A. Fréville et G. Plateau, The 0-1 bidimensional knapsack problem: Toward an efficient high-level primitive tool. J. Heuristics 2 (1997) 147-167.

[14] X. Gandibleux et A. Fréville, The multiobjective tabu search method customized on the 0/1 multiobjective knapsack problem: The two objectives case. J. Heuristics (à paraître).

[15] M. Garey et D. Johnson, Computers \& Intractability A Guide to the Theory of NPCompleteness. W.H. Freeman and Company (1979).

[16] B. Gavish et H. Pirkul, Allocation of data bases and processors in a distributed computting system. Management of Distributed Data Processing 31 (1982) 215-231.

[17] B. Gavish et H. Pirkul, Efficient algorithms for solving multiconstraint zero-one knapsack problems to optimality. Math. Programming 31 (1985) 78-105.

[18] P.C. Gilmore et R.E. Gomory, The theory and computation of knapsack functions. Oper. Res. 14 (1966) 1045-1074.

[19] F. Glover, Tabu search. ORSA J. Computing 2 (1990) 4-32.

[20] F. Glover et G.A. Kochenberger, Critical event tabu search for multidimensional knapsack problems, edité par I.H. Osman et J.P. Kelly, Metaheuristics: The Theory and Applications. Kluwer Academic Publishers (1996) 407-427.

[21] M. Gondran et M. Minoux, Graphes 83 algorithmes. Eyrolles (1985).

[22] S. Hanafi, A. El Abdellaoui et A. Fréville, Extension de la Méthode d'Élimination Inverse pour une gestion dynamique de la liste tabou. RAIRO (à paraître).

[23] S. Hanafi et A. Fréville, An efficient tabu search approach for the 0-1 multidimensional knapsack problem. Eur. J. Oper. Res. 106 (1998) 659-675.

[24] J.S. Lee et M. Guignard, An approximate algorithm for multidimensional zero-one knapsack problems a parametric approach. Management Sci. 34 (1998) 402-410.

[25] J. Lorie et L.J. Savage, Three problems in capital rationing. J. Business 28 (1955) 229-239.

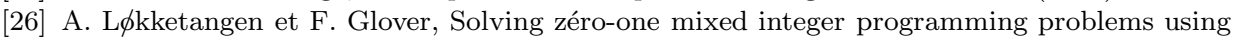
tabu search. Eur. J. Oper. Res. 106 (1998). Special Issue on Tabu Search.

[27] A. L $\phi k$ ketangen et F. Glover, Candidate list and exploration strategies for solving 0/1 mip problems using a pivot neighborhood, dans Metaheuristics. Kluwer Academic Publishers (1999).

[28] S. Martello et P. Toth, Knapsack Problems: Algorithms and Computer Implementations. John Wiley (1990).

[29] M.A. Osorio, F. Glover et P. Hammer, Cutting and surrogate constraint analysis for improved multidimensional knapsack solutions, Technical report. Hearin Center for Enterprise Science. Report HCES-08-00 (2000).

[30] W.H. Press, S.A. Teukolsky, W.T. Vetterling et B.P. Flannery, Numerical Recipes in C. Cambridge University Press (1992).

[31] W. Shih, A branch \& bound method for the multiconstraint zero-one knapsack problem. $J$. Oper. Res. Soc. 30 (1979) 369-378.

[32] Y. Toyoda, A simplified algorithm for obtaining approximate solutions to zero-one programming problem. Management Sci. 21 (1975) 1417-1427. 